\title{
Ventilação mecânica na doença pulmonar obstrutiva crônica e na asma
}

\author{
Mechanical ventilation in chronic obstructive pulmonary \\ disease and asthma
}

Sérgio da Cunha*

\section{Resumo}

As doenças pulmonares obstrutivas crônicas (DPOCs) e a asma brônquica caracterizam-se pela obstrução ao fluxo do ar nas vias aéreas, com consequente hiperinsuflação dinâmica. Quando não há resposta adequada à abordagem medicamentosa inicial torna-se necessária a assistência ventilatória mecânica. Sempre que possível lança-se mão de ventilação não invasiva (VNI), tendo como objetivo principal o repouso da musculatura inspiratória, ganhando-se tempo para a resposta ao tratamento dirigido à causa desencadeante. Para aqueles que não são candidatos à VNI, ou os que não melhoram após cerca de uma hora sob essa assistência, indica-se a ventilação mecânica invasiva. Os métodos ventilatórios mais frequentemente utilizados na ventilação mecânica invasiva são a ventilação com volume controlado (VCV) e a ventilação com pressão controlada (PCV). Não há estudos de grande porte demonstrando superioridade de uma sobre a outra na ventilação de pacientes com DPOC ou asma brônquica. Seja qual for o método utilizado, deve-se evitar o agravamento e tentar minimizar a hiperinsuflação alveolar. Para tanto, deve-se utilizar volume corrente e frequência respiratória baixos, assim como tempo expiratório longo. $\mathrm{O}$ uso de pressão positiva expiratória final (PEEP) extrínseca equivalente a até $85 \%$ da PEEP intrínseca pode ser útil em alguns casos para reduzir a hiperinsuflação alveolar.

Descritores: Doença pulmonar obstrutiva crônica; Asma; Respiração artificial; Ventilação não invasiva.
Abstract
Chronic obstructive pulmonary diseases (COPD) and asthma are characterized by ai- rways obstruction, with consequent dynamic hyperinflation. Mechanical ventilation is ne- cessary when there is no response to medical treatment. Noninvasive mechanical ventila- tion is used whenever possible, in which case inspiratory muscle rest is the main objective, 
during which period triggering factors treatment response is awaited. Invasive mechanical ventilation is indicated to those patients who are not candidates to noninvasive ventilation, or to those who do not get better after one hour of this ventilation method. Volume-controlled ventilation and pressure-controlled ventilation are the most frequently used invasive mechanical ventilation methods. There is no evidence that one of these methods is superior to the other in COPD or asthma patients' mechanical ventilation. Dynamic hyperinflation must be avoided and minimized whatever the ventilator method used. Low tidal volume and respiratory frequency, and high expiratory time must be used for this purpose. In selected cases, aiming alveolar hyperinflation reduction, extrinsic positive end expiratory pressure not above $85 \%$ of intrinsic positive end expiratory pressure may be used.

Keywords: Pulmonary disease, chronic obstructive; Asthma; Respiration; Artificial noninvasive ventilation.

\section{Ventilação mecânica na doença pulmonar obstrutiva crônica}

Sob a denominação de doença pulmonar obstrutiva crônica (DPOC) estão condições clínicas respiratórias que se caracterizam por obstrução crônica ao fluxo aéreo de caráter fixo ou parcialmente reversível, com graus variáveis de bronquite crônica e enfisema pulmonar. Não estão incluídas no grupo das DPOC: bronquiectasias difusas, sequelas de tuberculose, asma, bronquiolites, pneumoconioses ou outras doenças parenquimatosas pulmonares. ${ }^{1}$ Entre os critérios diagnósticos citam-se: tabagismo importante, tosse crônica, dispneia, broncoespasmo, gasometria arterial com hipoxemia e ou hipercapnia, radiografia de tórax com hiperinsuflação, e sobrecarga de câmaras cardíacas direitas. $^{2}$

A descompensação da DPOC está frequentemente associada a quadros infecciosos respiratórios. Também pode ocorrer por tromboem- bolismo pulmonar, pneumotórax espontâneo, arritmias cardíacas, uso de sedativos, ou por procedimentos cirúrgicos, especialmente os que envolvem o andar superior do abdome ou tórax.

A assistência ventilatória torna-se necessária mais frequentemente por hipoventilação alveolar(e consequente acidemia) do que por hipoxemia refratária à oferta de oxigênio. As principais indicações de ventilação mecânica na DPOC são: diminuição do nível de consciência, com perda da proteção das vias aéreas contra broncoaspiração; sinais clínicos de fadiga da musculatura inspiratória; acidose respiratória grave ( $\mathrm{pH}<7,25$ ); $\mathrm{PaCO}_{2}$ muito alta ocasionando arritmias cardíacas, instabilidade hemodinâmica ou sinais de edema cerebral. ${ }^{2}$

Havendo nível de consciência adequado e cooperação do paciente pode-se tentar, inicialmente, o suporte com ventilação não invasiva. ${ }^{3-5}$ Máscaras nasais, faciais ou faciais totais podem ser utilizadas. Utilizam-se pressões inspiratórias que garantam volume corrente de cerca de $7 \mathrm{ml} /$ kg peso teórico, e pressões expiratórias suficientes para reduzir a hiperinsuflação dinâmica, em geral entre 5 e $8 \mathrm{cmH}_{2} \mathrm{O}$. Períodos de ventilação não invasiva (VNI) de 40 a 50 minutos podem ser intercalados com oferta de oxigênio sob máscara de Hudson.Na VNI são mais frequentemente utilizadas a ventilação com suporte de pressão (PSV) associada à pressão positiva expiratória final (PEEP) e a ventilação com dois níveis de pressão (BIPAP). ${ }^{5}$

Para os casos mais graves, em especial aqueles em que não há melhora após 1 hora de VNI, ou há pneumonia extensa, queda do nível de consciência, incapacidade de mobilização de secreção respiratória, ou ainda os associados à instabilidade hemodinâmica, tornam-se necessárias a intubação orotraqueal (IOT) e a ventilação mecânica invasiva (VM).

Sempre que possível deve-se informar ao paciente e/ou aos seus familiares sobre a necessidade de IOT e VM. Monitorização de cardioscopia, oximetria de pulso e pressão arterial não invasiva devem preceder o procedimento, assim como o posicionamento adequado do 
paciente, na chamada posição olfativa, com o uso de coxim (cerca de $7 \mathrm{~cm}$ ) na região occipital e hiperextensão da cabeça. A verificação prévia de todos os materiais a serem utilizados é fundamental, entre eles: bolsa e máscara para ventilação acopladas à fonte de oxigênio, sistema de aspiração de secreções conectado e testado, laringoscópio com lâminas curva e reta testadas e adequadas ao paciente (lâminas de números 4 e 5 para adultos), estilete guia de intubação, tubos orotraqueais de diâmetros adequados (para adultos geralmente entre 8,0 e 9,5mm). Sedação e analgesia adequadas devem ser obtidas antes da IOT, em geral com o uso de midazolam $(0,1$ a $0,2 \mathrm{mg} / \mathrm{kg}$ ) ou propofol ( $1 \mathrm{mg} / \mathrm{kg})$, associado ao fentanil $(2 \mu \mathrm{g} / \mathrm{kg})$. Trata-se de medicamentos potentes, de rápido início de ação, e que requerem acompanhamento da pressão arterial, porque podem promover hipotensão arterial. $\mathrm{O}$ adequado relaxamento pode exigir o uso de bloqueadores neuromusculares, tais como a succinilcolina $(1 \mathrm{mg} / \mathrm{kg})$ ou o atracúrio $(0,5 \mathrm{mg} /$ $\mathrm{kg}$ ). Após a intubação e acoplamento ao ventilador deve-se elevar a cabeceira da cama 30 a 45', iniciar sedação e analgesia sob infusão venosa contínua, verificar a adequação da ventilação e oxigenação, e conferir o quadro hemodinâmico. Após essas providências iniciais, solicitar radiografia de controle da intubação, conferindo-se o posicionamento adequado da ponta do tubo (distante $2 \mathrm{~cm}$ da carina).

A escolha do método ventilatório e dos parâmetros ventilatórios desse método deve atender às especificidades da doença, que, como já mencionado, caracteriza-se por obstrução ao fluxo aéreo. A complicação a ser evitada é a hiperinsuflação dinâmica, e consequente auto-PEEP, com suas repercussões ventilatórias e hemodinâmicas (figura 1). Logo após a intubação traqueal recomenda-se período de 24 a 48 horas de repouso da musculatura inspiratória, o que pode ser obtido com as infusões de sedativo e analgésico. Método ventilatório ciclado a volume (ventilação ciclada a volume - VCV) ou método ciclado a tempo (ventilação com pressão controlada - PCV) pode ser utilizado na fase inicial da assistência ventilatória, não havendo estudo controlado de grande porte demonstrando superioridade de um sobre o outro na DPOC. ${ }^{1,5}$ Seja qual for o método selecionado, o importante é o ajuste do método para evitarmos hiperinsuflação dinâmica, volutrauma, barotrauma, atelectasia e toxicidade por oxigênio (quando usamos $\mathrm{FiO}_{2}>0,5$ ). Para tanto, alguns preconizam o conceito de hipoventilação controlada como estratégia ventilatória. Devemos prolongar o tempo expiratório, através de baixa frequência respiratória (10 a $14 \mathrm{rpm}$ )

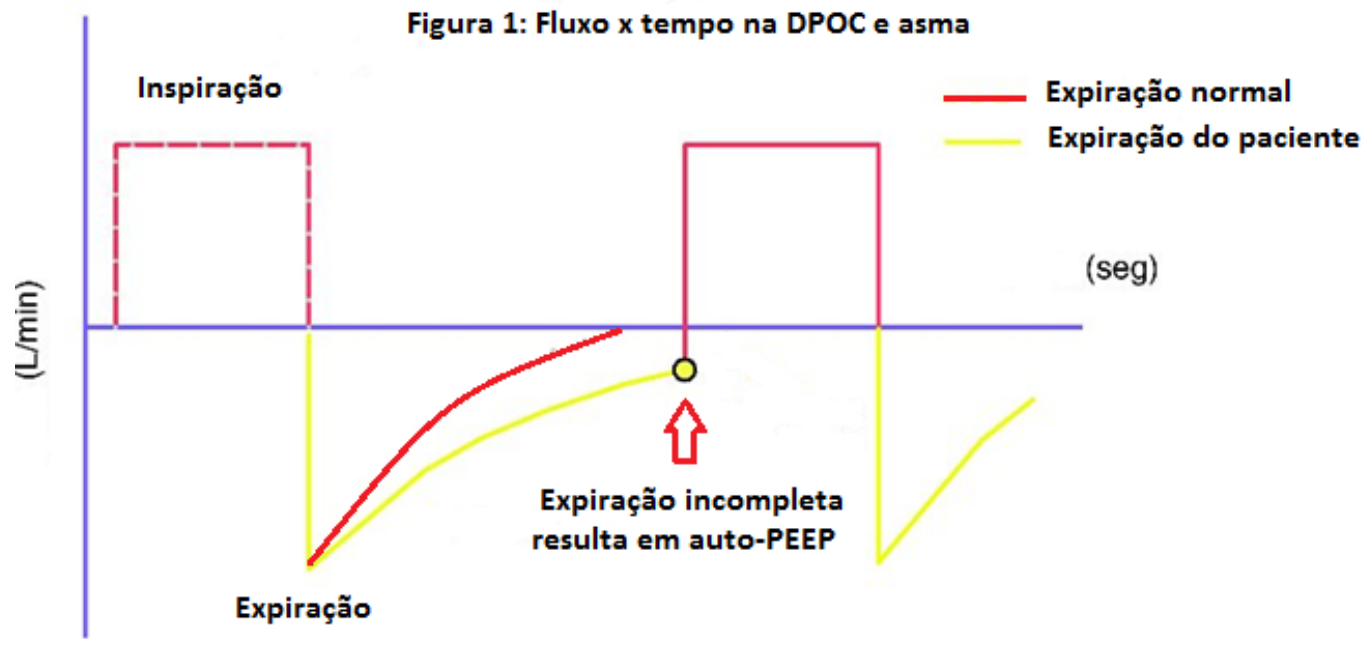

A obstrução ao fluxo expiratório resulta em prolongamento dessa fase do ciclo respiratório, levando ao aprisionamento de ar, com consequente auto-PEEP. A hiperinsuflação alveolar resultante desse processo é fator determinante de aumento de morbidade e mortalidade em pacientes com DPOC e asma submetidos à ventilação mecânica invasiva. 
e baixa relação inspiração:expiração (relação i:e < 1:3, isto é: 1:4, 1:5, etc). O volume corrente (VC) deve ser mantido entre 6 e $8 \mathrm{ml} / \mathrm{kg}$, de forma a manter volume minuto que resulte em pH sanguíneo entre 7,20 e 7,40, independente do nível de $\mathrm{PaCO}_{2} \cdot{ }^{1}$ Isto só não se aplica aos pacientes com arritmias secundárias à acidose, com hipertensão intracraniana e com síndromes coronarianas. Assim como em outras condições clínicas, a fração inspirada de oxigênio $\left(\mathrm{FiO}_{2}\right)$ deve ser a menor possível, que seja suficiente para manter a saturação arterial de oxigênio $\left(\mathrm{SaO}_{2}\right)>90 \%$. Na DPOC a PEEP extrínseca utilizada tem como objetivo a redução da hiperinsuflação dinâmica, não devendo ultrapassar 85\% do nível da auto-PEEP. A desinsuflação induzida pela PEEP extrínseca pode ser detectada pela queda da pressão de platô com o paciente no modo volume controlado, ou pelo aumento do volume corrente no modo pressão controlada. A sensibilidade do ventilador ao paciente deve ser ajustada em - 1 a $-2 \mathrm{cmH}_{2} \mathrm{O}$, quando controlada por variação de pressão no circuito, ou 21/min, quando por variação de fluxo. ${ }^{4}$

A PCV permite ventilação com menores pressões médias de vias aéreas, porém não garante VC. Variações na impedância do sistema respiratório promovem mudanças no VC que podem comprometer significativamente a ventilação, com consequente elevação da $\mathrm{PaCO}_{2}$ e acidose respiratória. Ajustes nos alarmes de volume minuto baixo e de frequência respiratória alta permitem um controle da limitação desse método ventilatório. A VCV, por sua vez, garante o VC até o limite de pressão estabelecido para prevenir o barotrauma, em geral não superior a $40 \mathrm{cmH}_{2} \mathrm{O}$ de pressão de pico, e 30 a $35 \mathrm{cmH}_{2} \mathrm{O}$ de pressão de platô, porém resulta em pressões médias de vias aéreas mais altas. Ajuste do alarme de pressão alta em vias aéreas, o estabelecimento de limite de pressão e o uso de volumes correntes baixos minimizam os inconvenientes da VCV.

Após os primeiros 20 minutos de ventilação podemos colher gasometria arterial visando à avaliação da adequação dos parâmetros ven- tilatórios. A necessidade de colheita frequente de gasometrias arteriais indica a instalação de cateter arterial, que também permite um melhor controle do quadro hemodinâmico.

Uma vez controlada a condição clínica desencadeadora da descompensação da DPOC, devemos reduzir gradativamente os níveis de sedação, visando à maior interação com o paciente e à mudança do método ventilatório. A ventilação com suporte pressórico (PSV) é frequentemente utilizada nessa fase. O nível de pressão de suporte deve ser o suficiente para manter o volume corrente preconizado (6 a $8 \mathrm{ml} /$ $\mathrm{kg}$ ), com frequência respiratória não superior a $30 \mathrm{rpm}$. Em geral, valores entre 15 e $20 \mathrm{cmH}_{2} 0$ são suficientes. $\mathrm{O}$ volume corrente gerado nesse método ventilatório é resultante não apenas do nível de pressão de suporte utilizado, mas também do trabalho da musculatura inspiratória do paciente e da impedância do sistema respiratório. A redução progressiva do nível de pressão de suporte induz a um aumento da participação da musculatura do paciente na sua ventilação alveolar. Para os pacientes mantidos por longos períodos em ventilação mecânica (> 21 dias) e com perda importante de massa muscular pode ser necessária a utilização de reduções programadas do nível do suporte pressórico, por curtos períodos, para melhor condicionamento da musculatura inspiratória (delta de PSV). Alguns pacientes com DPOC para os quais observamos dificuldade na redução do nível de pressão de suporte para níveis que permitam a descontinuação da ventilação mecânica podemos tentar o desmame em peça $\mathrm{T}$ progressiva. Isto se aplica apenas aos pacientes já submetidos à traqueostomia, que está indicada para aqueles que se mantém em ventilação mecânica por mais de 11 a 13 dias, sem expectativa de extubação em prazo curto (48 horas).

Nas primeiras 24 a 48 horas após a extubação podemos lançar mão de períodos de reexpansão pulmonar com VNI sob máscara facial (PSV + PEEP) ou BIPAP. Isto permite repouso da musculatura inspiratória e expansão 
pulmonar com maior mobilização de secreções respiratórias.

\section{Ventilação mecânica nas crises agudas de asma brônquica}

As principais indicações de ventilação mecânica nas crises de asma são: parada respiratória ou cardiorrespiratória; sinais de fadiga da musculatura inspiratória; hipoxemia $\left(\mathrm{PaO}_{2}<60 \mathrm{mmHg}\right.$ ou $\left.\mathrm{SaO}_{2}<90 \%\right)$ ou acidose respiratória; alteração do nível de consciência secundária às alterações dos gases sanguíneos (hipoxemia-agitação, hipercapnia-sedação). ${ }^{6}$

Os conceitos definidos na assistência ventilatória mecânica na DPOC aplicam-se aos pacientes com asma brônquica. Os valores propostos para os parâmetros ventilatórios na asma são ligeiramente diferentes dos preconizados para a DPOC, porém também têm como principal objetivo evitar o agravamento da hiperinsuflação alveolar. São os seguintes: VC entre 5 e 7 ml/kg; FR entre 7 e 11 rpm; pressão de pico inspiratório $<50 \mathrm{cmH}_{2} \mathrm{O}$; pressão de platô $<35 \mathrm{cmH}_{2} \mathrm{O}$; auto-PEEP $<15 \mathrm{cmH}_{2} \mathrm{O}$; PEEP extrínseca pode ser utilizada, com verificação do real benefício na redução da hiperinsuflação pulmonar. ${ }^{6}$ Em estudo experimental com coelhos visando avaliar o efeito da PEEP extrínseca $\left(5 \mathrm{cmH}_{2} \mathrm{O}\right)$ na distribuição do fluxo aéreo em animais normais comparados a animais nos quais induziu-se broncoespasmo pela administração de histamina, observou-se que a PEEP promoveu maior homogeneidade na distribuição do fluxo, com aumento do volume pulmonar. ${ }^{7}$

Medicamentos que promovem liberação de histamina, tais como morfina e meperidina, devem ser evitados, por agravarem o broncoespasmo. Os bloqueadores neuromusculares podem ser necessários para a adequada ventilação inicial de alguns pacientes, devendo ser interrompidos o mais rapidamente possível pela possibilidade de indução de neuromiopatia do paciente crítico, risco agravado pelo uso frequente de corticosteroides no tratamento da asma.

Para os casos mais resistentes, podemos lançar mão de agentes anestésicos inalatórios com propriedades broncodilatadoras.

$\mathrm{Na}$ análise de 127 pacientes com 160 crises de asma (status asmaticus) admitidos durante 10 anos em unidade intensiva americana observaram-se apenas 4 óbitos, todos relacionados a pacientes que haviam apresentado parada cardiorrespiratória fora do ambiente hospitalar, e que evoluíram para encefalopatia anóxica. ${ }^{8}$ Os autores apresentam como fatores de risco para mortalidade na asma os seguintes fatores: admissão hospitalar recente, episódios prévios de asma quase fatal, não adesão à medicação, acompanhamento extra-hospitalar ruim. Observou-se também que o percentual de pacientes admitidos em uso de corticosteroides inalatórios que era de $18 \%$ em 1990 havia mudado para $80 \%$ em 1998. Apenas 10 pacientes apresentaram complicações resultantes de barotrauma, o que foi atribuído ao uso de volumes correntes baixos.

Outo estudo americano avaliou pacientes admitidos em unidade intensiva por asma grave durante período de 30 anos. ${ }^{9}$ Duzentos e vinte e sete pacientes com 280 crises asmáticas foram avaliados. Faziam uso de corticosteroides $27 \%$ deles e $61 \%$ necessitaram de ventilação mecânica. O tempo de internação hospitalar foi semelhante entre os que necessitaram ou não de ventilação mecânica. A taxa de complicação foi baixa independente do uso de hipercapnia permissiva ou do modo ventilatório: mortalidade geral 0,4\%; pneumotórax 2,5\%; pneumonia 2,9\%. Observou-se redução da frequência de statusasmaticus de 12,4 para 3,2 casos por ano nos últimos 10 anos.

A mistura hélio-oxigênio (heliox) em substituição à tradicional, oxigênio-nitrogênio, tem a capacidade de reduzir a pressão resistiva na via aérea, reduzindo a turbulência do ar. Poucos estudos (e com número pequeno de pacientes) avaliaram este método. Isto também se aplica ao seu uso na DPOC. ${ }^{10,11}$

\section{Referências}

1. Jezler S, Holanda M, José A, et al. III Consenso Brasileiro de Ventilação Mecânica. Ventilação 
mecânica na doença pulmonar obstrutiva crônica (DPOC) descompensada. J Bras Pneumol. 2007;33(1):111-8.

2. Hospital Israelita Albert Einstein. Ventilação mecânica na DPOC. 2009; Disponível em: http:// medicalsuite.einstein.br/diretrizes/pneumol.

3. Rabbat A, Guetta A, Lorut C, et al. Management of acute exacerbations of COPD. Revue des maladies respiratoires. 2010 Oct;27(8):939-53.

4. Díaz Lobato S, Mayoralas Alises S, Montiel G. Noninvasive mechanical ventilation in the exacerbation of respiratory diseases. Medicina clínica. 2011 Dec 10;137(15):691-6.

5. García Vicente E, Sandoval Almengor JC, Díaz Caballero LA, et al. Invasive mechanical ventilation in COPD and asthma. Medicina intensiva / Sociedad Española de Medicina Intensiva y Unidades Coronarias. 2011;35(5):288-98.

6. Barbas C, Pinheiro B, Vianna A, et al. III Consenso Brasileiro de Ventilação Mecânica. Ventilação mecânica na crise de asma aguda. J Bras Pneumol. 2007;33(Supl 2):106-10.
7. Porra L, Suhonen H, Suortti P, et al. Effect of positive end-expiratory pressure on regional ventilation distribution during bronchoconstriction in rabbit studied by synchrotron radiation imaging. Crit. care med.]. 2011 Jul;39(7):1731-8.

8. Kao CC, Jain S, Guntupalli KK, et al. Mechanical ventilation for asthma: a 10-year experience. The Journal of asthma: official journal of the Association for the Care of Asthma. 2008 Sep;45(7):552-6.

9. Peters JI, Stupka JE, Singh H, et al. Status asthmaticus in the medical intensive care unit: a 30-year experience. Respiratory medicine. 2012 Mar;106(3):344-8.

10. Valli G, Paoletti P, Savi D, et al. Clinical use of Heliox in asthma and COPD. Monaldi archives for chest disease. 2007 Sep;67(3):159-64.

11. Diehl J-L, Peigne V, Guérot E, et al. Helium in the adult critical care setting. Annals of intensive care. 2011 Jan;1(1):24. 


\section{Autores}

\begin{abstract}
Abdiel Rolim
Programa de Residência Médica e Pós-graduação em Radiologia. Hospital Universitário Pedro Ernesto. Universidade do Estado do Rio de Janeiro. Rio de Janeiro, RJ, Brasil.
\end{abstract}

\section{Adalgisa I. M. Bromerschenckel}

Programa de Pós-graduação Stricto Sensu em Ciências Médicas. Faculdade de Ciências Médicas. Universidade do Estado do Rio de Janeiro. Rio de Janeiro, RJ, Brasil.

\section{Agnaldo José Lopes}

Disciplina de Pneumologia e Tisiologia. Departamento de Especialidades Médicas. Faculdade de Ciências Médicas. Universidade do Estado do Rio de Janeiro. Rio de Janeiro, RJ, Brasil.

\section{Ana Paula V. Soares}

Serviço de Pneumologia e Tisiologia. Policlínica Piquet Carneiro. Universidade do Estado do Rio de Janeiro. Rio de Janeiro, RJ, Brasil.

\section{Anamelia C. Faria}

Serviço de Pneumologia e Tisiologia. Hospital Universitário Pedro Ernesto. Universidade do Estado do Rio de Janeiro. Rio de Janeiro, RJ, Brasil.

\section{Domenico Capone}

Disciplina de Pneumologia e Tisiologia. Departamento de Especialidades Médicas. Faculdade de Ciências Médicas. Universidade do Estado do Rio de Janeiro. Rio de Janeiro, RJ, Brasil.

\section{Eduardo Costa F. Silva}

Serviço de Alergia e Imunologia. Departamento de Medicina Interna. Hospital Universitário Pedro Ernesto. Universidade do Estado do Rio de Janeiro. Rio de Janeiro, RJ, Brasil.

\section{Elizabeth J. C. Bessa}

Disciplina de Pneumologia e Tisiologia. Faculdade de Ciências Médicas. Universidade do Estado do Rio de Janeiro. Rio de Janeiro, RJ, Brasil.

\section{Gabriela A. C. Dias}

Serviço de Alergia e Imunologia. Departamento de Medicina Interna. Hospital Universitário Pedro Ernesto. Universidade do Estado do Rio de Janeiro. Rio de Janeiro, RJ, Brasil.

\section{Jorge Eduardo Pio}

Disciplina de Pneumologia e Tisiologia. Faculdade de Ciências Médicas. Universidade do Estado do Rio de Janeiro. Rio de Janeiro, RJ, Brasil.

\section{Kênia M. da Silva}

Programa de Pós-graduação Stricto Sensu em Ciências Médicas. Faculdade de Ciências Médicas. Universidade do Estado do Rio de Janeiro. Rio de Janeiro, RJ, Brasil.

\section{Leonardo P. Bruno}

Serviço de Pneumologia e Tisiologia. Hospital Universitário Pedro Ernesto. Universidade do Estado do Rio de Janeiro. Rio de Janeiro, RJ, Brasil.

\section{Lívia I. de O. Souza}

Faculdade de Ciências Médicas. Universidade do Estado do Rio de Janeiro. Rio de Janeiro, RJ, Brasil.

\section{Mateus Bettencourt}

Programa de Pós-graduação Stricto Sensu em Ciências Médicas. Faculdade de Ciências Médicas. Universidade do Estado do Rio de Janeiro. Rio de Janeiro, RJ, Brasil.

\section{Paulo Roberto Chauvet}

Disciplina de Pneumologia e Tisiologia. Faculdade de Ciências Médicas. Universidade do Estado do Rio de Janeiro. Rio de Janeiro, RJ, Brasil. 


\section{Rafael Capone}

Programa de Residência Médica e Pós-graduação em Radiologia. Hospital Universitário Pedro Ernesto. Universidade do Estado do Rio de Janeiro. Rio de Janeiro, RJ, Brasil.

\section{Renato Azambuja}

Serviço de Pneumologia e Tisiologia. Hospital Universitário Pedro Ernesto. Universidade do Estado do Rio de Janeiro. Rio de Janeiro, RJ, Brasil.

\section{Rogério M. Bártholo}

Programa de Pós-graduação Stricto Sensu em Ciências Médicas. Faculdade de Ciências Médicas. Universidade do Estado do Rio de Janeiro. Rio de Janeiro, RJ, Brasil.

\section{Sérgio da Cunha}

Disciplina de Tratamento Intensivo. Departamento de Clínica Médica. Faculdade de Ciências Médicas. Universidade do Estado do Rio de Janeiro. Rio de Janeiro, RJ, Brasil.

\section{Thiago P. Bártholo}

Serviço de Pneumologia e Tisiologia. Hospital Universitário Pedro Ernesto. Universidade do Estado do Rio de Janeiro. Rio de Janeiro, RJ, Brasil.

\section{Verônica S. Câmara}

Serviço de Pneumologia e Tisiologia. Policlínica Piquet Carneiro. Universidade do Estado do Rio de Janeiro. Rio de Janeiro, RJ, Brasil. 